\title{
TRANSCENDENCE OF SPECIAL VALUES OF POCHHAMMER FUNCTIONS
}

\author{
MARVIN D. TRETKOFF AND PAULA TRETKOFF
}

\begin{abstract}
In this paper, we examine the set of algebraic numbers at which higher order hypergeometric functions take algebraic values. In particular, we deduce criteria for this set to be finite and for it to be infinite.
\end{abstract}

\section{INTRODUCTION}

The exceptional set of a transcendental function of one complex variable is the set of algebraic numbers at which it assumes algebraic values. This set plays a fundamental role in the theory of transcendental numbers. For example, the transcendence of $e$ and $\pi$ are immediate consequences of the classical results of Hermite (1873), Lindemann (1882) and Weierstrass (1885) that the exceptional set of the exponential function, $\exp (z)$, consists only of $z=0$.

In the present paper, we characterize the exceptional set of $m$-th order Pochhammer functions, $m>3$, in terms of certain members of a family of abelian varieties. We establish criteria for this set to be finite and for it to be infinite.

The Pochhammer functions are solutions of $m$-th order ordinary differential equations of Fuchsian class with $m+1$ regular singular points. They were introduced by Pochhammer and, independently, by Tissot. When $m=2$, these functions are the classical hypergeometric functions $w=F(a, b, c ; z)$, defined by the differential equation

$$
z(1-z) w^{\prime \prime}+(c-(a+b+1) z) w^{\prime}-a b w=0,
$$

where $a, b, c$ are given constants. Higher order Pochhammer functions arise by fixing all but one of the variables in Appell-Lauricella's hypergeometric functions of $n \geq 2$ complex variables.

The task of investigating the exceptional set of the hypergeometric function $F(a, b, c ; z)$, with $a, b, c$ rational numbers, was posed by C.L. Siegel in 1929. Despite significant advances in transcendental number theory during the intervening time, Siegel's problem remained unsolved for almost sixty

Typeset January 16, 2008 [18:34].

1991 Mathematics Subject Classification. 11J91, 33C65.

Key words and phrases. Hypergeometric functions, transcendence, Shimura varieties. 
years. Then, in 1988, J. Wolfart [27] made a pioneering breakthrough: he associated a family of abelian varieties to each triple $(a, b, c)$ and showed that the exceptional set corresponds to certain members of this family that admit complex multiplication. Wolfart applied this correspondence to obtain conditions on $(a, b, c)$ that imply that the exceptional set is infinite. Using Wolfart's description, P. Cohen and G. Wüstholz [8] found conditions on $(a, b, c)$ that imply that this set is finite. Their results were conditional on a particular case of the André-Oort Conjecture that was subsequently proved in a deep paper of S. Edixhoven and A. Yafaev [16]. Thus, Siegel's problem was completely solved: necessary and sufficient conditions were obtained, in terms of $(a, b, c)$, for the exceptional set of $F(a, b, c ; z)$ to be finite.

A description of the exceptional set of third order Pochhamer functions is the main result obtained in [14]. Once again, a family of abelian varieties is associated to each such function. However, in sharp contrast to the case of the classical hypergeometric functions, not all the abelian varieties corresponding to the exceptional set admit complex multiplication. In fact, those admitting complex multiplication form a proper subset of the totality that is defined by subtle conditions.

In the present paper, we pursue this line of investigation for Pochhammer functions of arbitrary order $m$. For $m>3$, we find even more possibilities for the abelian varieties corresponding to the exceptional set. This leads to more involved criteria for the finiteness of this set. We also obtain results that yield refinements of [14] when specialized to the case $m=3$.

The plan of the paper is as follows. In Section 2, we define the Pochhammer functions. We construct an analytic family of abelian varieties associated to these functions in Section 3. In Section 4, we use transcendence techniques to describe the exceptional set of the Pochhammer function in terms of certain isogeny classes of abelian varieties. In Section 5, we assume these abelian varieties share a common factor with complex multiplication, and we deduce criteria for the exceptional set to be infinite in this case. In Sections 6 and 7 we obtain criteria for the finiteness of the exceptional set. Finally, under the hypothesis of Section 5, the results in Section 7 are shown to yield necessary and sufficient conditions for finiteness. Thus, we obtain the analogue of the solution to Siegel's problem.

\section{Pochhammer Functions}

In this section, we introduce the functions studied by Pochhammer in his investigation of $(n+1)$-st order ordinary differential equations whose singular points are all regular and are $n+2$ in number. Pochhammer's work was apparently anticipated by Tissot. For more details see [21]. The main results of this paper concern the transcendence of values of these Pochhammer functions at algebraic numbers. These results generalize results about the Gauss hypergeometric function, $n=1$, (see, [8], [27]) and about a function studied by Picard, $n=2$, (see, [12], [14]). 
Let $\left\{\mu_{i}\right\}_{i=0}^{n+2}$ be rational numbers satisfying the conditions

$$
0<\mu_{i}<1, \quad i=0, \ldots, n+2, \quad \sum_{i=0}^{n+2} \mu_{i}=2 .
$$

(This is a ball $(n+3)$-tuple in the sense of [9].) We refer to such numbers as rational $(n+3)$-tuples.

Let,

$(2.2) \mathcal{Q}_{n}=\left\{\mathbf{x}=\left(x_{2}, \ldots, x_{n+1}\right) \in \mathbb{P}_{1}(\mathbb{C})^{n}: x_{i} \neq 0,1, \infty ; \quad x_{i} \neq x_{j}, i \neq j\right\}$.

For $\mathbf{x} \in \mathcal{Q}_{n}$, let $\omega_{1}$ be the differential form

$$
\omega_{1}=\omega_{1}(\mu ; \mathbf{x})=u^{-\mu_{0}}(u-1)^{-\mu_{1}} \prod_{i=2}^{n+1}\left(u-x_{i}\right)^{-\mu_{i}} d u .
$$

Suppose from now on that $\mu_{0}+\mu_{n+1}<1$ and let $\mu^{\prime}=\left\{\mu_{i}^{\prime}\right\}_{i=0}^{n+1}$ be the rational $(n+2)$-tuple with

$$
\mu_{0}^{\prime}=\mu_{0}+\mu_{n+1}, \quad \mu_{n+1}^{\prime}=\mu_{n+2}, \quad \mu_{i}^{\prime}=\mu_{i}, \quad i=1, \ldots, n .
$$

For a fixed $\boldsymbol{\Lambda}=\left(\lambda_{2}, \ldots, \lambda_{n}\right) \in \mathcal{Q}_{n-1}$, we define the Pochhammer function of a single variable $y \in \mathbb{C} \backslash\left\{0,1, \lambda_{2}, \ldots, \lambda_{n}\right\}$ by

$$
\Phi_{\mu, \boldsymbol{\Lambda}}=\Phi_{\mu, \boldsymbol{\Lambda}}(y)=\int_{1}^{\infty} \omega_{1}(\mu ; \boldsymbol{\Lambda}, y) / \int_{1}^{\infty} \omega_{1}\left(\mu^{\prime} ; \boldsymbol{\Lambda}\right) .
$$

Notice that $\Phi_{\mu, \Lambda}(0)=1$.

For $\boldsymbol{\Lambda} \in \mathcal{Q}_{n-1}$, we define the exceptional set $\mathcal{E}_{\mu, \boldsymbol{\Lambda}}$ of $\Phi_{\mu, \boldsymbol{\Lambda}}$ to be

$$
\mathcal{E}_{\mu, \boldsymbol{\Lambda}}=\left\{y \in \overline{\mathbb{Q}} \backslash\left\{0,1, \lambda_{2}, \ldots, \lambda_{n}\right\}: \Phi_{\mu, \boldsymbol{\Lambda}}(y) \in \overline{\mathbb{Q}}^{*}\right\} .
$$

The main focus of this paper is the study of the set $\mathcal{E}_{\mu, \boldsymbol{\Lambda}}$.

The function $\Phi_{\mu, \boldsymbol{\Lambda}}$ satisfies a linear ordinary differential equation of order $n+1$, called the Tissot-Pochhammer differential equation. We refer the reader to [21] for the theory of this equation. It has $n+2$ regular singular points at $y=0,1, \lambda_{2}, \ldots, \lambda_{n}, \infty$. A basis of solutions at a regular singular point is given by $\int_{g}^{h} \omega(\mu ; \boldsymbol{\Lambda}, y)$ for certain $g \neq h$ in $\left\{0,1, \lambda_{2}, \ldots, \lambda_{n}, \infty\right\}$.

\section{Abelian varieties}

In this section, we associate an analytic family of abelian varieties to the Pochhammer function. Related constructions occur in [2],[6],[7],[24].

Let $\mu=\left\{\mu_{i}\right\}_{i=0}^{n+2}$ be a rational $(n+3)$-tuple. Let $N$ be the least common denominator of the $\mu_{i}$, and let $K=\mathbb{Q}(\zeta)$, where $\zeta=\exp (2 \pi i / N)$. For $s \in(\mathbb{Z} / N \mathbb{Z})^{*}$, let $\sigma_{s}$ be the Galois embedding of $K$ which maps $\zeta \mapsto \zeta^{s}$.

For $\mathbf{x} \in \mathcal{Q}_{n}, n \geq 1$, and $f$ a divisor of $N$, let $\mathcal{C}_{\mu, f}=\mathcal{C}_{\mu, f}(\mathbf{x})$ be the projective non-singular curve with affine model

$$
w^{f}=u^{N \mu_{0}}(u-1)^{N \mu_{1}} \prod_{i=2}^{n+1}\left(u-x_{i}\right)^{N \mu_{i}} .
$$


For $\mathbf{x} \in \overline{\mathbb{Q}}^{n}$, this curve is defined over a number field.

To every $\mathbf{x} \in \mathcal{Q}_{n}$, we can associate an abelian variety $T_{\mu, N}=T_{\mu, N}(\mathbf{x})$ as follows. For each proper divisor $f$ of $N$, there is a natural surjection of Jacobians from $\operatorname{Jac}\left(\mathcal{C}_{\mu, N}(\mathbf{x})\right)$ onto $\operatorname{Jac}\left(\mathcal{C}_{\mu, f}(\mathbf{x})\right)$. Let $T_{\mu, N}=T_{\mu, N}(\mathbf{x})$ be the connected component of the origin in the intersection of all the kernels of these epimorphisms. The automorphism $\chi:(u, w) \mapsto\left(u, \zeta^{-1} w\right)$ of the affine model of $\mathcal{C}_{\mu, N}$ induces an action of $\zeta$ on $T_{\mu, N}$, which realizes the field $K$ in its endomorphism algebra $\operatorname{End}_{0}\left(T_{\mu, N}\right)=\operatorname{End}\left(T_{\mu, N}\right) \otimes \mathbb{Q}$, and an action $\chi^{*}$ of $K$ on the space of differentials of the first kind $H^{0}\left(T_{\mu, N}, \Omega\right)$ on $T_{\mu, N}$. For $s \in(\mathbb{Z} / N \mathbb{Z})^{*}$, let $V_{s}$ be the subspace of the elements of $H^{0}\left(T_{\mu, N}, \Omega\right)$ on which $\chi^{*}(K)$ acts by multiplication by $\sigma_{s}(K)$. The dimension of $V_{s}$ is given by,

$$
r_{s}=r_{s}^{(\mu)}=-1+\sum_{i=0}^{n+2}\left\langle s \mu_{i}\right\rangle,
$$

(see, for example, [4]), where $\langle x\rangle$ denotes the fractional part of a real number $x$. For all $s \in(\mathbb{Z} / N \mathbb{Z})^{*}$, we have $r_{s}+r_{-s}=n+1$. The space $H^{0}\left(T_{\mu, N}, \Omega\right)$ is the direct sum of the $V_{s}$; therefore the dimension of $T_{\mu, N}$ is $(n+1) \varphi(N) / 2$. We say that $T_{\mu, N}$ has generalized complex multiplication with type

$$
\Psi=\Psi_{\mu}=\sum_{s \in(\mathbb{Z} / N \mathbb{Z})^{*}} r_{s} \sigma_{s}
$$

Notice that $r_{1}=1$. The space $V_{1}$ is generated by the differential form on $T_{\mu, N}$ corresponding to $\omega_{1}$, as defined by $(2.3)$.

The complex abelian variety $T_{\mu, N}=T_{\mu, N}(\mathbf{x})$ has the structure of a polarized complex torus. Replacing $T_{\mu, N}$ by an isogenous abelian variety if necessary, we can assume that the complex torus has the form $\mathbb{C}^{d} / \mathcal{M}$, $d=\operatorname{dim}_{\mathbb{C}}\left(T_{\mu, N}\right)$, with $\mathcal{M}=\mathbb{Z}[\zeta]^{n+1}$, and that the polarization is given, independently of $\mathrm{x} \in \mathcal{Q}_{n}$, by a matrix $T \in M_{n+1}(K)$, with $\bar{T}^{t}=-T$ and $\operatorname{tr}_{\mathbb{K} / \mathbb{Q}}(\mathcal{M} T \overline{\mathcal{M}}) \subseteq \mathbb{Z}$. The datum $\left(K, \Psi, \mathbb{Z}[\zeta]^{n+1}, T\right)$ determines a Shimura variety $S$ of dimension

$$
\operatorname{dim}(S)=\sum_{s \in(\mathbb{Z} / N \mathbb{Z})^{*} /\{ \pm 1\}} r_{s} r_{-s}
$$

with the $r_{s}$ as in (3.1). The points of $S(\mathbb{C})$ form a complex analytic space given by the quotient of a complex symmetric domain $\mathcal{H}(K, \Psi)$ by an arithmetic group $\Gamma(\mathcal{M}, T)$. We have,

$$
\mathcal{H}(K, \Psi)=\prod_{s \in(\mathbb{Z} / N \mathbb{Z})^{*} /\{ \pm 1\}} \mathcal{H}_{r_{s}, r_{-s}}
$$

where, for $u v \neq 0$,

$$
\mathcal{H}_{u, v}=\left\{z \in M_{u, v}(\mathbb{C}): 1-z^{t} \bar{z} \quad \text { positive and hermitian }\right\},
$$

and $\mathcal{H}_{u, v}$ is a point for $u v=0$. When $s=1$, we have $r_{1}=1, r_{-1}=n$ and $\mathcal{H}_{1, n}$ is the complex $n$-ball $\mathbb{B}_{n}$. See [25] for more details. 
There is an analytic multi-valued map from $\mathcal{Q}_{n}$ to $\mathcal{H}(K, \Psi)$ whose image in $S(\mathbb{C})$ associates to each $\mathbf{x} \in \mathcal{Q}_{n}$ the unique point $P_{\mathbf{x}} \in S(\mathbb{C})$ representing the isomorphism class of the polarized abelian variety $T_{\mu, N}(\mathbf{x})$. The point $P_{\mathbf{x}}$ corresponds to a single $\Gamma(\mathcal{M}, T)$-orbit in $\mathcal{H}(K, \Psi)$. For $\boldsymbol{\Lambda} \in \mathcal{Q}_{n-1}$, let $U_{\boldsymbol{\Lambda}}=\left\{P_{\boldsymbol{\Lambda}, y}:(\boldsymbol{\Lambda}, y) \in \mathcal{Q}_{n}\right\}$. The analytic space $S$ can be compactified to a complex projective variety $\bar{S}$. By the extension theorem for analytic sets (see [17], p181), the closure $\overline{U_{\boldsymbol{\Lambda}}}$ of $U_{\boldsymbol{\Lambda}}$ in the strong topology of $\bar{S}$ is a closed analytic subset of complex dimension one. By Chow's theorem (see $[22]), \overline{U_{\boldsymbol{\Lambda}}}$ has the structure of an algebraic variety. Let $\mathcal{C}_{\boldsymbol{\Lambda}}$ be the complex algebraic curve in $S$ given by $\overline{U_{\Lambda}} \cap S$.

\section{Transcendence arguments}

The results of this section generalize to arbitrary $n \geq 1$ the transcendence arguments of [8], [27], for $n=1$, and of [12],[14], for $n=2$.

For two non-zero complex numbers $a, b$, we write $a \sim b$ if $a / b$ is algebraic. We then say that $a$ and $b$ are proportional over $\overline{\mathbb{Q}}$.

For two abelian varieties $A$ and $B$, we write $A \widehat{=} B$ when $A$ and $B$ are isogenous. For an abelian variety $C$ defined over $\overline{\mathbb{Q}}$, let $H^{0}\left(C, \Omega_{\overline{\mathbb{Q}}}\right)$ be the space of differential forms of the first kind on $C$, defined over $\overline{\mathbb{Q}}$. Denote by $\mathcal{P}_{C}$ the $\overline{\mathbb{Q}}$-vector space generated by the numbers,

$$
\left\{\int_{\gamma} \omega: \quad \omega \in H^{0}\left(C ; \Omega_{\overline{\mathbb{Q}}}\right) ; \quad \gamma \in H_{1}(C ; \mathbb{Z})\right\} .
$$

If the endomorphism algebra $\operatorname{End}_{0}(C)$ of $C$ contains a number field $F$, then we say that $C$ is $F$-stable. If $C$ is $F$-stable and contains no proper non-trivial $F$-stable abelian subvariety, we say that $C$ is $F$-irreducible. Otherwise, we say that $C$ is $F$-reducible. If $F=K=\mathbb{Q}(\zeta)$, let $V_{C, s}$ be the eigenspace of $H^{0}(C, \Omega)$ on which $K$ acts by multiplication by $\sigma_{s}(K)$.

We begin with the following corollary of Theorem 5 in [29] (for more details see [24], Prop. 1, p.6 and [23], Appendix).

Proposition 4.1. Let $A$ and $B$ be abelian varieties defined over $\overline{\mathbb{Q}}$. Then $\mathcal{P}_{A} \cap \mathcal{P}_{B} \neq\{0\}$ if and only if there exist non-trivial simple abelian subvarieties $A^{\prime}$ of $A$, and $B^{\prime}$ of $B$, such that $A^{\prime} \widehat{=} B^{\prime}$.

We apply this result to the two abelian varieties $A=T_{\mu, N}(\boldsymbol{\Lambda}, y)$ and $B=T_{\mu^{\prime}, N}(\boldsymbol{\Lambda})$, for $\boldsymbol{\Lambda} \in \mathcal{Q}_{n-1} \cap \overline{\mathbb{Q}}^{n-1}$, with $\int_{1}^{\infty} \omega_{1}\left(\mu^{\prime}, \boldsymbol{\Lambda}\right) \neq 0$, and $y \in \mathcal{E}_{\mu, \boldsymbol{\Lambda}}$ (for notation, see Sections 2 and 3). By (2.5), as $y \in \mathcal{E}_{\mu, \boldsymbol{\Lambda}}$, we have the relation

$$
\int_{1}^{\infty} \omega_{1}(\mu ; \boldsymbol{\Lambda}, y) \sim \int_{1}^{\infty} \omega_{1}\left(\mu^{\prime} ; \mathbf{\Lambda}\right)
$$


between non-zero numbers. If $\gamma$ is the Pochhammer cycle between 1 and $\infty$, this implies the relation

$$
\int_{\gamma} \omega_{1}(\mu ; \boldsymbol{\Lambda}, y) \sim \int_{\gamma} \omega_{1}\left(\mu^{\prime} ; \boldsymbol{\Lambda}\right)
$$

between non-zero numbers, so that $\mathcal{P}_{T_{\mu, N}(\boldsymbol{\Lambda}, y)} \cap \mathcal{P}_{T_{\mu^{\prime}, N}(\boldsymbol{\Lambda})}$ is non-trivial and contains a non-zero period of $\omega_{1}\left(\mu^{\prime} ; \boldsymbol{\Lambda}\right)$. Therefore, by Proposition 4.1, $T_{\mu, N}(\boldsymbol{\Lambda}, y)$ and $T_{\mu^{\prime}, N}(\boldsymbol{\Lambda})$ share a non-trivial simple factor $E$ up to isogeny. The isogeny class of $E$ is independent of $y \in \mathcal{E}_{\mu, \boldsymbol{\Lambda}}$. Applying [3], Section 1, Example 3 to this situation, we deduce that there are decompositions

$$
T_{\mu^{\prime}, N}(\boldsymbol{\Lambda}) \hat{=} E^{u} \times C, \quad T_{\mu, N}(\boldsymbol{\Lambda}, y) \widehat{=} E^{u} \times D,
$$

where $E^{u}, C$, and $D$ are $K$-stable abelian varieties (for an appropriate choice of $u$ ) with $V_{C, 1}=V_{D, 1}=\{0\}$. Moreover, if $T_{\mu^{\prime}, N}(\boldsymbol{\Lambda})$ is $K$ irreducible, then the abelian variety $C$ is trivial. Since

$$
\operatorname{dim}\left(T_{\mu, N}(\boldsymbol{\Lambda}, y)\right)=\operatorname{dim}\left(T_{\mu^{\prime}, N}(\boldsymbol{\Lambda})\right)+\frac{1}{2} \varphi(N),
$$

we must in that case have $\operatorname{dim}(D)=\frac{1}{2} \varphi(N)$. Therefore $D$ is of CM type and $T_{\mu, N}(\boldsymbol{\Lambda}, y) \widehat{=} T_{\mu^{\prime}, N}(\boldsymbol{\Lambda}) \times D$.

We summarize the above discussion by the following theorem.

Theorem 4.2. Let $\boldsymbol{\Lambda} \in \mathcal{Q}_{n-1} \cap \overline{\mathbb{Q}}^{n-1}$ with $\int_{1}^{\infty} \omega_{1}\left(\mu^{\prime}, \boldsymbol{\Lambda}\right) \neq 0$. There are fixed $K$-stable abelian varieties $A_{1}$ and $A_{2}$ with

$$
T_{\mu^{\prime}, N}(\boldsymbol{\Lambda}) \hat{=} A_{1} \times A_{2},
$$

where $A_{1}$ is $K$-irreducible and $V_{A_{2}, 1}=\{0\}$. Suppose that $y \in \mathcal{E}_{\mu, \boldsymbol{\Lambda}}$. Then

$$
T_{\mu, N}(\boldsymbol{\Lambda}, y) \hat{=} A_{1} \times D,
$$

where $D$ is a $K$-stable abelian variety with $V_{D, 1}=\{0\}$. The isogeny class of $A_{1}$ is independent of $y \in \mathcal{E}_{\mu, \boldsymbol{\Lambda}}$. If $A_{2}$ is trivial, then $D$ is of CM type with isogeny class independent of $y$ : we call this the irreducible case.

Let $\boldsymbol{\Lambda} \in \mathcal{Q}_{n-1}$ satisfy the conditions of Theorem 4.2. In the irreducible case, the points of the exceptional set $\mathcal{E}_{\mu, \Lambda}$ of the Pochhammer function correspond to abelian varieties $T_{\mu, N}(\boldsymbol{\Lambda}, y)$ in a fixed isogeny class represented by $T_{\mu^{\prime}, N}(\boldsymbol{\Lambda}) \times D$. Otherwise, the isogeny class of the abelian variety $D$ may vary with $y \in \mathcal{E}_{\mu, \Lambda}$.

\section{Conditions for the exceptional set to Be infinite}

In this section we present some sufficient conditions for the exceptional set to be infinite, thereby generalizing to $n>2$ results of [8],[27], for $n=1$, and [12],[14], for $n=2$. We show that, for certain $\Lambda \in \mathcal{Q}_{n-1} \cap \overline{\mathbb{Q}}^{n-1}$, the exceptional set $\mathcal{E}_{\mu, \boldsymbol{\Lambda}}$ is infinite when the curve $\mathcal{C}_{\boldsymbol{\Lambda}}$ of $\S 3$ is weakly special in $S$. Consider Shimura morphisms $\varphi_{1}: T \rightarrow S, \varphi_{2}: T \rightarrow T^{\prime}$ and a point $t^{\prime} \in T^{\prime}$. An irreducible component of $\varphi_{1}\left(\varphi_{2}^{-1}\left(t^{\prime}\right)\right)$, or of its image under a 
Hecke operator, is called a weakly special subvariety of $S$. For a detailed discussion of weakly special subvarieties see [18].

We have the following partial converse of Theorem 4.2:

Theorem 5.1. For $n \geq 1$ and $(\boldsymbol{\Lambda}, y) \in \mathcal{Q}_{n} \cap \overline{\mathbb{Q}}^{n}$, suppose that both $\int_{1}^{\infty} \omega_{1}\left(\mu^{\prime} ; \boldsymbol{\Lambda}\right)$ and $\int_{1}^{\infty} \omega_{1}(\mu ; \boldsymbol{\Lambda}, y)$ are non-zero. Let $A_{1}$ be a $K$-stable abelian variety of $C M$ type with $\operatorname{dim}\left(V_{A_{1}, 1}\right) \neq 0$. If there are isogenies of the form

$$
T_{\mu^{\prime}, N}(\boldsymbol{\Lambda}) \hat{=} A_{1} \times A_{2}, \quad T_{\mu, N}(\boldsymbol{\Lambda}, y) \hat{=} A_{1} \times D
$$

for abelian varieties $A_{2}$ and $D$, then

$$
\int_{1}^{\infty} \omega_{1}\left(\mu^{\prime} ; \boldsymbol{\Lambda}\right) \sim \int_{1}^{\infty} \omega_{1}(\mu ; \boldsymbol{\Lambda}, y) .
$$

Proof: By (2.1) and (2.4), the vector space $V_{A_{1}, 1}$ is 1-dimensional. Let $\omega_{1} \in H^{0}\left(A_{1}, \Omega\right)$ be a generator of this space. As $A_{1}$ is of CM type, the nonzero periods of $\omega_{1}$ are all proportional over $\overline{\mathbb{Q}}$. Moreover, the isogenies in (5.1) induce $K$-equivariant maps on differential 1 -forms that send the differential forms corresponding to $\omega_{1}\left(\mu^{\prime} ; \boldsymbol{\Lambda}\right)$ and $\omega_{1}(\mu ; \boldsymbol{\Lambda}, y)$ to multiples of $\omega_{1}$ by a nonzero algebraic number. Let $\gamma$ be the Pochhammer cycle between 1 and $\infty$. The numbers $\int_{\gamma} \omega_{1}\left(\mu^{\prime} ; \boldsymbol{\Lambda}\right)$ and $\int_{\gamma} \omega_{1}(\mu ; \boldsymbol{\Lambda}, y)$ are therefore proportional over $\overline{\mathbb{Q}}$ to periods of $\omega_{1}$. All the periods of $\omega_{1}$ are proportional over $\overline{\mathbb{Q}}$, so that $\int_{\gamma} \omega_{1}\left(\mu^{\prime} ; \boldsymbol{\Lambda}\right)$ and $\int_{\gamma} \omega_{1}(\mu ; \boldsymbol{\Lambda}, y)$ are proportional over $\overline{\mathbb{Q}}$. Replacing the Pochhammer cycle by the line integral from 1 to $\infty$ multiplies these periods by an element of $\overline{\mathbb{Q}}^{*}$, so that (5.2) follows.

Theorem 5.2. Let $\boldsymbol{\Lambda} \in \mathcal{Q}_{n-1} \cap \overline{\mathbb{Q}}^{n-1}$ with $\int_{1}^{\infty} \omega_{1}\left(\mu^{\prime}, \boldsymbol{\Lambda}\right) \neq 0$. Suppose that $T_{\mu^{\prime}, N}(\boldsymbol{\Lambda}) \hat{=} A_{1} \times A_{2}$ where $A_{1}$ is $K$-stable, of CM type, with $\operatorname{dim}\left(V_{A_{1}, 1}\right) \neq 0$. Suppose that $\mathcal{E}_{\mu, \boldsymbol{\Lambda}}$ is not empty. If $\mathcal{C}_{\boldsymbol{\Lambda}}$ is a weakly special curve in $S$, then $\mathcal{E}_{\mu, \Lambda}$ is infinite.

Proof: By Theorem 4.2 and Theorem 5.1 the exceptional set $\mathcal{E}_{\mu, \boldsymbol{\Lambda}}$ is a subset of finite complement in the set of $y \in \overline{\mathbb{Q}}$, with $(\boldsymbol{\Lambda}, y) \in \mathcal{Q}_{n}$, and such that $T_{\mu, N}(\boldsymbol{\Lambda}, y)$ contains up to isogeny the abelian variety $A_{1}$ as a factor. If $\mathcal{C}_{\boldsymbol{\Lambda}}$ is a weakly special curve, once there is one such $y$ there are infinitely many, so that $\mathcal{E}_{\mu, \Lambda}$ is also infinite.

\section{Conditions for the EXCeptional Set to BE Finite}

We assume throughout this section that $\Lambda \in \mathcal{Q}_{n-1} \cap \overline{\mathbb{Q}}^{n-1}$ and that $\int_{1}^{\infty} \omega_{1}\left(\mu^{\prime}, \boldsymbol{\Lambda}\right) \neq 0$. By Theorem 4.2 , there are fixed $K$-stable abelian varieties $A_{1}$ and $A_{2}$ such that, for all $y \in \mathcal{E}_{\mu, \Lambda}$,

$$
T_{\mu^{\prime}, N}(\boldsymbol{\Lambda}) \hat{=} A_{1} \times A_{2}, \quad T_{\mu, N}(\boldsymbol{\Lambda}, y) \widehat{=} A_{1} \times D,
$$

where $D$ is a $K$-stable abelian variety with $V_{D, 1}=\{0\}$. In the irreducible case, the abelian variety $A_{2}$ is trivial and $D$ lies in a fixed isogeny class of CM type. In general, it is possible that $D$ may vary with $y$. 
Denote by $\Psi_{D}$ the generalized CM type of $D$ and by $\Psi_{1}$ the generalized CM type of $A_{1}$. With $\Psi_{\mu}$ as in Section 3, we have from (6.1),

$$
\Psi_{\mu}=\Psi_{1}+\Psi_{D}=\sum_{s \in(\mathbb{Z} / N \mathbb{Z})^{*}} r_{s}^{(\mu)} \sigma_{s} .
$$

Write

$$
\Psi_{1}=\sum_{s \in(\mathbb{Z} / N \mathbb{Z})^{*}} r_{s}^{(1)} \sigma_{s}, \quad \Psi_{D}=\sum_{s \in(\mathbb{Z} / N \mathbb{Z})^{*}} r_{s}^{(D)} \sigma_{s}
$$

Then $r_{s}^{(1)}+r_{-s}^{(1)}=m_{1} \geq 1$ and $r_{s}^{(D)}+r_{-s}^{(D)}=m_{D} \geq 1$ where $m_{1}+m_{D}=n+1$. We have $r_{1}^{(D)}=0$. Moreover, if $A_{2}$ is non-trivial, then $m_{1} \leq n-1$. Let

(6.4) $\operatorname{dim}_{1}=\sum_{s \in(\mathbb{Z} / N \mathbb{Z})^{*} /\{ \pm 1\}} r_{s}^{(1)} r_{-s}^{(1)}, \quad \operatorname{dim}_{D}=\sum_{s \in(\mathbb{Z} / N \mathbb{Z})^{*} /\{ \pm 1\}} r_{s}^{(D)} r_{-s}^{(D)}$

Let $\mathcal{C}_{\boldsymbol{\Lambda}} \subset S$ be as in Section 3. From Theorem 4.2, we deduce the following.

Theorem 6.1. Let $\boldsymbol{\Lambda} \in \mathcal{Q}_{n-1} \cap \overline{\mathbb{Q}}^{n-1}$ with $\int_{1}^{\infty} \omega_{1}\left(\mu^{\prime}, \boldsymbol{\Lambda}\right) \neq 0$. Suppose that $\mathcal{C}_{\boldsymbol{\Lambda}}$ has finite intersection with the union of all special subvarieties of $S$ of dimension at most $\operatorname{dim}_{1}+\operatorname{dim}_{D}$. Then $\mathcal{E}_{\mu, \boldsymbol{\Lambda}}$ is finite.

Let $S_{\boldsymbol{\Lambda}}$ be the smallest special subvariety of $S$ containing $\mathcal{C}_{\boldsymbol{\Lambda}}$. A conjecture of Pink [19] implies that the hypothesis of Theorem 6.1 is true provided

$$
\operatorname{dim}\left(S_{\boldsymbol{\Lambda}}\right)>\operatorname{dim}_{1}+\operatorname{dim}_{D}+1 .
$$

When $T_{\mu^{\prime}, N}(\boldsymbol{\Lambda})$ is $K$-irreducible, we have $\operatorname{dim}_{D}=0$. If, in addition, $A_{1}$ is of CM type, then $\operatorname{dim}_{1}=0$ and we can in fact deduce from Theorem 7.2 that $\mathcal{E}_{\mu, \boldsymbol{\Lambda}}$ is finite if $\mathcal{C}_{\boldsymbol{\Lambda}}$ is not special, see $\S 7$.

Proposition 6.2. Let $\boldsymbol{\Lambda} \in \mathcal{Q}_{n-1}$. The inequality (6.5):

$$
\operatorname{dim}\left(S_{\boldsymbol{\Lambda}}\right)>\operatorname{dim}_{1}+\operatorname{dim}_{D}+1,
$$

is satisfied provided $n \geq 2, m_{1} \leq n-1$, and

$$
\operatorname{dim}\left(S_{\boldsymbol{\Lambda}}\right)>\operatorname{dim}(S)+m_{1}-n .
$$

In particular, (6.5) is satisfied provided $n \geq 2, T_{\mu^{\prime}, N}(\boldsymbol{\Lambda})$ is $K$-reducible and $\operatorname{dim}\left(S_{\boldsymbol{\Lambda}}\right)=\operatorname{dim}(S)$.

Proof: We have

$$
\operatorname{dim}(S)=\sum_{s \in(\mathbb{Z} / N \mathbb{Z})^{*} /\{ \pm 1\}} r_{s}^{(\mu)} r_{-s}^{(\mu)}
$$

and

(6.7) $\operatorname{dim}_{1}=\sum_{s \in(\mathbb{Z} / N \mathbb{Z})^{*} /\{ \pm 1\}} r_{s}^{(1)} r_{-s}^{(1)}, \quad \operatorname{dim}_{D}=\sum_{s \in(\mathbb{Z} / N \mathbb{Z})^{*} /\{ \pm 1\}} r_{s}^{(D)} r_{-s}^{(D)}$ 
where $r_{s}^{(\mu)}=r_{s}^{(1)}+r_{s}^{(D)}$ for all $s \in(\mathbb{Z} / N \mathbb{Z})^{*}$, and $r_{1}^{(\mu)}=1, r_{1}^{(1)}=1$, $r_{1}^{(D)}=0$. Therefore

$$
r_{1}^{(\mu)} r_{-1}^{(\mu)}=n, \quad r_{1}^{(1)} r_{-1}^{(1)}=m_{1}-1, \quad r_{1}^{(D)} r_{-1}^{(D)}=0,
$$

and, for each $s \in(\mathbb{Z} / N \mathbb{Z})^{*}$,

$$
r_{s}^{(\mu)} r_{-s}^{(\mu)}=\left(r_{s}^{(1)}+r_{s}^{(D)}\right)\left(r_{-s}^{(1)}+r_{-s}^{(D)}\right) \geq r_{s}^{(1)} r_{-s}^{(1)}+r_{s}^{(D)} r_{-s}^{(D)} .
$$

From (6.7) and (6.9) we deduce that $\operatorname{dim}_{1}+\operatorname{dim}_{D}+1$ is not greater than

$$
m_{1}+\sum_{s \neq 1, s \in(\mathbb{Z} / N \mathbb{Z})^{*} /\{ \pm 1\}} r_{s}^{(\mu)} r_{-s}^{(\mu)}=\operatorname{dim}(S)+m_{1}-n .
$$

Combining (6.10) with the hypothesis of the proposition, we have

$$
\operatorname{dim}\left(S_{\boldsymbol{\Lambda}}\right)>\operatorname{dim}(S)+m_{1}-n \geq \operatorname{dim}_{1}+\operatorname{dim}_{D}+1
$$

as required.

By Theorem 5.2, Pink's conjecture implies that, if $\mathcal{E}_{\mu, \Lambda}$ is not empty, if $\mathcal{C}_{\boldsymbol{\Lambda}}$ is weakly special, and if $A_{1}$ is of CM type, we must have

$$
\operatorname{dim}\left(S_{\boldsymbol{\Lambda}}\right) \leq \operatorname{dim}_{D}+1 .
$$

\section{ANother FIniteness CRITERION IN THE IRREDUCIBle CASE}

Throughout this section, we assume that $\Lambda \in \mathcal{Q}_{n-1} \cap \overline{\mathbb{Q}}^{n-1}$ satisfies $\int_{1}^{\infty} \omega_{1}\left(\mu^{\prime}, \boldsymbol{\Lambda}\right) \neq 0$, and that the abelian variety $T_{\mu^{\prime}, N}(\boldsymbol{\Lambda})$ is $K$-irreducible. By Theorem 4.2, the exceptional $\mathcal{E}_{\mu, \boldsymbol{\Lambda}}$ is then a subset of the set,

$$
\overline{\mathcal{E}}_{\mu, \boldsymbol{\Lambda}}=\left\{y \in \overline{\mathbb{Q}}: \quad(\boldsymbol{\Lambda}, y) \in \mathcal{Q}_{n} \quad \text { and } \quad T_{\mu, N}(\boldsymbol{\Lambda}, y) \widehat{=} T_{\mu^{\prime}, N}(\boldsymbol{\Lambda}) \times D\right\},
$$

where $D$ is a fixed abelian variety of CM type. We therefore have:

Theorem 7.1. Let $\boldsymbol{\Lambda} \in \mathcal{Q}_{n-1} \cap \overline{\mathbb{Q}}^{n-1}$ with $\int_{1}^{\infty} \omega_{1}\left(\mu^{\prime}, \boldsymbol{\Lambda}\right) \neq 0$ and $T_{\mu^{\prime}, N}(\boldsymbol{\Lambda})$ $K$-irreducible. Let $\Omega \subset S$ be the set of moduli of $S$ corresponding to abelian varieties in the same isogeny class as $T_{\mu^{\prime}, N}(\boldsymbol{\Lambda}) \times D$. If $\mathcal{C}_{\boldsymbol{\Lambda}} \cap \Omega$ is finite, then $\mathcal{E}_{\mu, \boldsymbol{\Lambda}}$ is finite.

Notice that Theorem 5.2 is a partial converse to this theorem. A weakly special curve containing a $\mathrm{CM}$ point is a special curve. When $T_{\mu^{\prime}, N}(\boldsymbol{\Lambda})$ is a $K$-irreducible abelian variety with complex multiplication, and $\mathcal{C}_{\boldsymbol{\Lambda}}$ is a weakly special curve, it follows that $\mathcal{C}_{\boldsymbol{\Lambda}}$ is a special curve if $\mathcal{E}_{\mu, \boldsymbol{\Lambda}}$ is not empty. Combining the particular case of the André-Oort Conjecture proved in [16] and Theorem 5.2, we have the following result.

Theorem 7.2. Let $\boldsymbol{\Lambda} \in \mathcal{Q}_{n-1} \cap \overline{\mathbb{Q}}^{n-1}$ with $\int_{1}^{\infty} \omega_{1}\left(\mu^{\prime}, \boldsymbol{\Lambda}\right) \neq 0$. Suppose that $T_{\mu^{\prime}, N}(\boldsymbol{\Lambda})$ is $K$-irreducible and of CM type. Assume that $\mathcal{E}_{\mu, \boldsymbol{\Lambda}}$ is not empty. Then $\mathcal{E}_{\mu, \boldsymbol{\Lambda}}$ is a finite set if and only if $\mathcal{C}_{\boldsymbol{\Lambda}}$ is not a special curve in $S$.

When $n=1$, Theorem 7.2 gives the results of [8],[27].

A conjecture of Pink [18] asserts that, under the hypotheses of Theorem 7.1, the intersection $\mathcal{C}_{\boldsymbol{\Lambda}} \cap \Omega$ is finite if $\mathcal{C}_{\boldsymbol{\Lambda}}$ is not a weakly special curve. 


\section{REFERENCES}

[1] Appell, P., Kampé de Fériet, M.J.: Fonctions hypergéométriques et hypersphériques. Polynômes d'Hermite, Gauthier-Villars 1926.

[2] Archinard, N.: Hypergeometric abelian varieties. Canad. J. Math. 55 (5) (2003), 897932.

[3] Bertrand, D.: Endomorphismes de groupes algébriques; applications arithmétiques. In: Approximations diophantiennes et nombres transcendants, Prog. Math. 31, 1983, $1-45$.

[4] Chevalley, Cl., Weil, A.: Uber das Verhalten der Integrale 1. Gattung bei Automorphismen des Funktionkörpers. Abh. Hamburger Math. Sem. 10 (1934), 358-361.

[5] Cohen, P.B.: Humbert surfaces and transcendence properties of automorphic functions. Rocky Mountain J. Math., 26 (3) (1996), 987-1001.

[6] Cohen, P.B., Wolfart, J: Modular embeddings for some non-arithmetic Fuchsian groups. Acta Arithmetica, LVI (1990), 93-110.

[7] Cohen, P.B., Wolfart, J.: Fonctions hypergéométriques en plusieurs variables et espaces des modules de variétés abéliennes. Ann. scient. Éc. Norm. Sup., 4e série, t.26, (1993), 665-690.

[8] Cohen, P.B., Wüstholz, G: Application of the André-Oort conjecture to some questions in transcendence. In: Panorama of Number Theory, A view from Baker's garden (ed. by G. Wüstholz), Cambridge U. Press, Cambridge 2002, 89-106.

[9] Deligne, P., Mostow, G.D.: Monodromy of hypergeometric functions and nonlattice integral monodromy. Publ. I.H.E.S. 63 (1986), 5-89.

[10] Desrousseaux, P-A.: Valeurs exceptionnelles de fonctions hypergéométriques d'Appell. Ramanujan J. 8 (3) (2004), 331-355.

[11] Desrousseaux, P-A: Fonctions hypergéométriques de Lauricella, périodes de variétés abéliennes et transcendance. Comptes rendus mathém. de l'Acad. des Sciences, La Soc. royale de Canada 26 (4) (2004), 110-117.

[12] Desrousseaux, P-A: Valeurs d'une fonction de Picard en des points algébriques, J. of Number Theory 125 (2007), 95-116.

[13] Desrousseaux, P-A: Exceptional sets of Lauricella hypergeometric functions, Journal of Algebra, Number Theory and Applications 5 (3), 429-467.

[14] Desrousseaux, P-A, Tretkoff, M.D., Tretkoff, P.: Transcendence of values at algebraic points for certain higher-order hypergeometric functions. IMRN (61) (2005), 38353854 .

[15] Desrousseaux, P-A, Tretkoff, M.D., Tretkoff, P.: Zariski-density of exceptional sets for hypergeometric functions, preprint 2005, to appear in Forum Mathematicum.

[16] Edixhoven, S., Yafaev, A: Subvarieties of Shimura varieties. Annals of Math. 157 (2003), 621-645.

[17] Grauert, H., Remmert, R.: Coherent analytic sheaves, Grundlehren math. Wissen. 265, Springer (1984).

[18] Pink, R.: A Combination of the Conjectures by Mordell-Lang and André-Oort. In: Geometric Methods in Algebra and Number Theory (Bogomolov, F., Tschinkel, Y., Eds.) Progress in Math., 235, Basel: Birkhäuser (2005), 251-282.

[19] Pink, R.: A Common Generalization of the Conjectures of André-Oort. ManinMumford, and Mordell-Lang, preprint, April 17, 2005.

[20] Pochhammer, L.: Ueber hypergeometrische Funktionen $n^{\text {ter }}$ Ordnung, J. f. die reine und angewandte Mathematik, 71, 316-352.

[21] Schlesinger, L.: Handbuch der Theorie der Linearen Differentialgleichungen, Zweiter Band, Teubner, Stuttgart 1897; reprinted by Johnson Reprint Corp., New York, 1968.

[22] Serre, J-P.: Géométrie algébrique et géométrie analytique, Annales de l'Institut Fourier 6 (1956), 1-42. 
[23] Shiga, H., Tsutsui, T., Wolfart, J.: Triangle Fuchsian differential equations with apparent singularities (with an appendix by P. B. Cohen). Osaka J. Math. 41 (2004), 625-658.

[24] Shiga, H., Wolfart, J: Criteria for complex multiplication and transcendence properties of automorphic functions. J. Reine Angew. Math. 463 (1995), 1-25.

[25] Shimura, G: On analytic families of polarized abelian varieties and automorphic functions. Annals of Math. 78 (1963), 149-192.

[26] Shimura, G., Taniyama, Y: Complex multiplication of abelian varieties and its applications to number theory. Publ. Math. Soc. Japan 6, 1961.

[27] Wolfart, J: Werte hypergeometrische Funktionen. Invent. Math. 92 (1988), 187-216.

[28] Wüstholz, G.: Zum Periodenproblem. Invent. Math. 78 (1984), 381-391.

[29] Wüstholz, G.: Algebraic groups, Hodge theory and transcendence. In: Proc. of the Intern. Congress of Math. 1, Berkeley, California, USA, 1986, 476-483.

[30] Yoshida, M: Fuchsian differential equations. Aspects of math., Vieweg, 1987.

Current address: Department of Mathematics, Texas A\&M University, TAMU 3368, TX 77843-3368

E-mail address: ptretkoff@math.tamu.edu 